\title{
Delay-Dependent Stability Conditions for Non-autonomous Functional Differential Equations with Several Delays in a Banach Space
}

https://doi.org/10.1515/msds-2020-0132

Received July 23, 2020; accepted May 4, 2021

Abstract: Let $B_{j}(t)(j=1, \ldots, m)$ and $B(t, \tau)(t \geq 0,0 \leq \tau \leq 1)$ be bounded variable operators in a Banach space. We consider the equation

$$
u^{\prime}(t)=\sum_{k=1}^{m} B_{k}(t) u\left(t-h_{k}(t)\right)+\int_{0}^{1} B(t, \tau) u\left(t-h_{0}(\tau)\right) d \tau(t \geq 0),
$$

where $h_{k}(t)(t \geq 0 ; k=1, \ldots, m)$ and $h_{0}(\tau)$ are continuous nonnegative bounded functions. Explicit delay-dependent exponential stability conditions for that equation are established. Applications to integrodifferential equations with delay are also discussed.

Keywords: differential-delay equations in a Banach space; stability; integro-differential equations

MSC: 34K30, 34K06, 34K20

\section{Introduction and statement of the main result}

This paper is devoted to a class of linear nonautonomous functional differential equations in a Banach space with several variable delays, whose coefficients are bounded operators. Such equations include integrodifferential equations with delay.

The basic method for the stability analysis of functional differential equations is the Lyapunov-Krasovskij method [5]. By that method, many great results have been obtained. Recently, that method has been extended to functional differential equations in a Hilbert space, cf. $[10,11,14,16,18]$ and references given therein. Besides, mainly equations with one delay have been considered. To the best of our knowledge, the stability of nonautonomous equations in a Banach space with several delays are not investigated in the available literature. Below we obtain delay-dependent exponential stability conditions for nonautonomous functionaldifferential equations in a Banach space with several delays.

It should be noted that finding the Lyapunov-Krasovskij type functionals or solving the corresponding operator inequalities are often connected with serious mathematical difficulties, especially regarding nonautonomous equations with many delays. To the contrary, the stability conditions presented in this paper are explicitly formulated in terms of the coefficients and delays. The literature on the delay-dependent stability criteria is rather rich, but mainly equations in a finite dimensional space are considered, cf. [7, 8, 15].

^Corresponding Author: Michael Gil’: Department of Mathematics, Ben Gurion University of the Negev, P.0. Box 653, BeerSheva 84105, Israel, E-mail: gilmi@bezeqint.net 
Introduce the notations. Everywhere below, $X$ is a complex Banach space with a norm $\|\cdot\| x=\|\cdot\|$ and the unit operator $I_{X}=I$. By $\mathcal{B}(X)$, we denote the set of all bounded linear operators in $X$. For an $A \in \mathcal{B}(X)$, $\sigma(A)$ is the spectrum and $\|A\|$ is the operator norm.

For real $a<b<\infty, C([a, b], X)$ is the space of $X$-valued functions $f$ defined and continuous on $[a, b]$, and equipped with the finite norm

$$
\|f\|_{C(a, b)}=\|f\|_{C([a, b], X)}=\sup _{a \leq t \leq b}\|f(t)\|_{X},
$$

and $C([a, \infty), X)$ is the space of $X$-valued functions $f$ defined, bounded and continuous on $[a, \infty)$ with the finite norm $\|f\|_{C(a, \infty)}=\|f\|_{C([a, \infty), x)}=\sup _{t \geq a}\|f(t)\|_{x}$. Put $R_{+}=[0, \infty)$ and $R_{\eta}=[-\eta, \infty)$ with $0<\eta<\infty$, and introduce on $C\left(R_{\eta}, X\right)$ the operator $E$ by

$$
(E u)(t)=\sum_{k=1}^{m} B_{k}(t) u\left(t-h_{k}(t)\right)+\int_{0}^{1} B(t, \tau) u\left(t-h_{0}(\tau)\right) d \tau \quad\left(u \in C\left(R_{\eta}, X\right) ; t \geq 0\right),
$$

where $h_{k}(t) \quad(k=1, \ldots, m)$ and $h_{0}(\tau)$ are continuous nonnegative functions defined on $R_{+}$and $[0,1]$, respectively, such that

$$
\sup _{t \geq 0} h_{k}(t) \leq \eta \quad(k=1, \ldots, m) \text { and } \sup _{0 \leq \tau \leq 1} h_{0}(\tau) \leq \eta ;
$$

$B_{j}(t): R_{+} \rightarrow \mathcal{B}(X)(j=1, \ldots, m)$ are continuous in the operator norm, $B(t, \tau): R_{+} \times[0,1] \rightarrow \mathcal{B}(X)$ is continuous in the operator norm in $t$ and piece-wise continuous in $\tau$.

The present paper is devoted to the equation

$$
d v(t) / d t=(E v)(t)(t \geq 0)
$$

with the initial condition

$$
v(t)=\phi(t) \quad(-\eta \leq t \leq 0, \phi \in C([-\eta, 0], x)),
$$

where $\phi$ is given. A solution of problem (1.1), (1.2) is a function $v: R_{\eta} \rightarrow X$ having a strong derivative $v^{\prime}(t) \in X$ for each finite $t>0$ and satisfying (1.1) and (1.2).

Below we check the existence of solutions to (1.1) under consideration. Equation (1.1) is said to be exponentially stable if there are positive constants $m_{0}, \delta_{0}$ independent of the initial function, such that

$$
\|v(t)\| x \leq\|\phi\|_{C([-\eta, 0], x)} m_{0} e^{-\delta_{0} t}(t \geq 0)
$$

for any solution $v(t)$ of problem (1.1), (1.2).

Throughout the paper it is a assumed that

$$
\chi_{0}:=\sup _{t \geq 0}\left(\sum_{k=1}^{m}\left\|B_{k}(t)\right\|+\int_{0}^{1}\|B(t, \tau)\| d \tau\right)<\infty
$$

and therefore,

$$
\psi_{0}:=\sup _{t \geq 0} \sum_{k=1}^{m}\left\|B_{k}(t)\right\| h_{k}(t)+\int_{0}^{1}\|B(t, \tau)\| h_{0}(\tau) d \tau<\infty .
$$

Put

$$
M(t):=\sum_{k=1}^{m} B_{k}(t)+\int_{0}^{1} B(t, \tau) d \tau
$$

and introduce the operator $V_{M}$ by

$$
\left(V_{M} f\right)(t)=\int_{0}^{t} U(t, s) f(s) d s \quad\left(f \in C\left(R_{+}, X\right)\right)
$$


where $U(t, s)$ is the evolution operator of the differential equation

$$
z^{\prime}(t)=M(t) z(t)
$$

Now we are in a position to formulate our main result.

Theorem 1.1. Let $V_{M}$ be bounded in $C\left(R_{+}, X\right)$ with a norm $\left\|V_{M}\right\|_{C\left(R_{+}, X\right)}$. Let the conditions (1.3) and

$$
\left\|V_{M}\right\|_{C\left(R_{+}, x\right)} \chi_{0} \psi_{0}<1
$$

hold. Then equation (1.1) is exponentially stable.

The proof of this theorem is presented in the next section.

As it follows from Theorem 1.1 our stability conditions are based, in particular, on the norm estimates for $V_{M}$. Below we consider such estimates under various conditions. In particular, in Section 3 we assume that $M(t)$ is dissipative. In Section 4 it is supposed that $M(t)$ satisfied the so called generalized Lipschitz condition. Section 5 is devoted to equations with differentiable operators a Hilbert space.

In Section 6 Theorem 1.1 is applied to integro-differential equations.

\section{Proof of Theorem 1.1}

Consider the non-homogeneous equation corresponding to (1.1):

$$
w^{\prime}(t)=(E w)(t)+f(t) \quad\left(f \in C\left(R_{+}\right)=C\left(R_{+}, X\right), t \geq 0\right)
$$

with the zero initial condition

$$
w(t)=0, t \leq 0 .
$$

A solution of problem (2.1), (2.2) is a strongly differentiable function $w: R_{\eta} \rightarrow X$ satisfying (2.1) for all finite $t>0$ and (2.2). Integrating (2.1), we have

$$
w(t)=\int_{0}^{t}(E w)(s) d s+f_{1}(t),
$$

where

$$
f_{1}(t)=\int_{0}^{t} f(s) d s .
$$

For each finite $T>0$ the operator $W$ defined in space $C(0, T)=C([0, T], X)$ by

$$
(W f)(t)=\int_{0}^{t}(E f)(s) d s \quad(f \in C(0, T))
$$

is a Volterra one and therefore, problemg (2.1), (2.2) has a unique solution representable by

$$
w=\sum_{k=0}^{\infty} W^{k} f_{1} .
$$

Furthermore, for a finite $T>0$ for the brevity put $|w|_{T}=\|w\|_{C(0, T)}$. Due to (1.3) for a solution of (2.1), (2.2) we have.

$$
|E w|_{T} \leq \chi_{0}|w|_{T} .
$$

Now (2.1) implies

$$
\left|w^{\prime}\right|_{T} \leq \chi_{0}|w|_{T}+\|f\|_{C\left(R_{+}\right)}(0<T<\infty) .
$$


We have $(E w)(t)=M(t) w(t)+(Z w)(t)(t \geq 0)$, where

$$
(Z w)(t)=\sum_{k=1}^{m} B_{k}(t)\left(w\left(t-h_{k}(t)\right)-w(t)\right)+\int_{0}^{1} B(t, \tau)\left(w\left(t-h_{0}(\tau)\right)-w(t)\right) d \tau .
$$

Thus (2.1) can be written as

$$
w^{\prime}(t)=M(t) w(t)+(Z w)(t)+f(t) \quad(t \geq 0) .
$$

By the Variation of Constants formula problem (2.5), (2.2) takes the form

$$
w(t)=\int_{0}^{t} U(t, s)((Z w)(s)+f(s)) d s .
$$

Observe that

$$
w(t)-w(t-\tau)=\int_{t-\tau}^{t} w^{\prime}(s) d s(t \geq 0, \tau>0) .
$$

Hence, by (2.4)

$$
\|w(t)-w(t-\tau)\|_{C(0, T)} \leq \tau\left|w^{\prime}\right|_{T} \leq \chi_{0} \tau|w|_{T}+\tau\|f\|_{C\left(R_{+}\right)} .
$$

Thus

$$
\begin{gathered}
|Z w|_{T} \leq \sup _{0 \leq t \leq T}\left(\sum_{k=1}^{m}\left\|B_{k}(t)\right\|\left\|w\left(t-h_{k}(t)\right)-w(t)\right\|+\int_{0}^{1}\|B(t, \tau)\|\left\|w\left(t-h_{0}(\tau)\right)-w(t)\right\| d \tau\right) \\
\quad \leq \sup _{t \geq 0}\left(\sum_{k=1}^{m}\left\|B_{k}(t)\right\| h_{k}(t)\left(\chi_{0}|w|_{T}+\|f\|_{C\left(R_{+}\right)}\right)+\int_{0}^{1}\|B(t, \tau)\| h_{0}(\tau)\left(\chi_{0}|w|_{T}+\|f\|_{C\left(R_{+}\right)}\right) d \tau\right) .
\end{gathered}
$$

Therefore,

$$
|Z w|_{T} \leq \psi_{0}\left(\chi_{0}|w|_{T}+\|f\|_{C\left(R_{+}\right)}\right)
$$

From (2.6) and (2.8) it follows

$$
\begin{gathered}
|w|_{T} \leq\left\|V_{M}\right\|_{C(0, T)}\|Z w+f\|_{C(0, T)} \leq\left\|V_{M}\right\|_{C(0, T)}\left(|(Z w)|_{T}+\|f\|_{C\left(R_{+}\right)}\right) \\
\leq\left\|V_{M}\right\|_{C(0, T)}\left(\psi_{0} \chi_{0}|w|_{T}+\left(\psi_{0}+1\right)\|f\|_{C\left(R_{+}\right)}\right) .
\end{gathered}
$$

If (1.5) holds, then

$$
|w|_{T} \leq\left(1-\chi_{0} \psi_{0}\left\|V_{M}\right\|_{C\left(R_{+}\right)}\right)^{-1}\left(\psi_{0}+1\right)\left\|V_{M}\right\|_{C\left(R_{+}\right)}\|f\|_{C\left(R_{+}\right)} .
$$

Hence, letting $T \rightarrow \infty$, we get

$$
\|w\|_{C\left(R_{+}\right)} \leq\left(1-\chi_{0} \psi_{0}\left\|V_{M}\right\|_{C\left(R_{+}\right)}\right)^{-1}\left(\psi_{0}+1\right)\left\|V_{M}\right\|_{C\left(R_{+}\right)}\|f\|_{C\left(R_{+}\right)} .
$$

We thus have proved the following result.

Lemma 2.1. Under the hypothesis of Theorem 1.1, for any $f \in C\left(R_{+}\right)$problem (2.1), (2.2) has a unique solution $w$, which satisfies inequality (2.9).

Let $v(t)$ be a solution of problem (1.1), (1.2). Put

$$
\hat{\phi}(t)= \begin{cases}\phi(0) & \text { if } t \geq 0 \\ \phi(t) & \text { if }-\eta \leq t<0\end{cases}
$$

and $x_{0}(t)=v(t)-\hat{\phi}(t)$. Since $d \hat{\phi}(t) / d t=0 \quad(t \geq 0)$, from (1.1) it follows

$$
x_{0}^{\prime}(t)=\left(E x_{0}\right)(t)+f_{0}(t) \quad(t>0)
$$


where $f_{0}=E \hat{\phi}$. Besides, the zero initial condition holds. Under conditions (1.3), (1.5) Lemma 2.1 implies

$$
\left.\left\|x_{0}\right\|_{C\left(R_{+}\right)} \leq\left(1-\psi_{0} \chi_{0}\left\|V_{M}\right\|_{C\left(R_{+}\right)}\right)^{-1}\left(1+\psi_{0}\right)\left\|V_{M}\right\|_{C\left(R_{+}\right)}\left\|f_{0}\right\|_{C\left(R_{+}\right)}\right) .
$$

Note that $\|\hat{\phi}\|_{C\left(R_{+}\right)} \leq\|\phi\|_{C(-\eta, 0)}$ and due to (2.3)

$$
\left\|f_{0}\right\|_{C\left(R_{+}\right)} \leq \chi_{0}\|\hat{\phi}\|_{C\left(R_{+}\right)} \leq \chi_{0}\|\phi\|_{C(-\eta, 0)} .
$$

Since,

$$
\|v\|_{C\left(R_{+}\right)} \leq\left\|x_{0}\right\|_{C\left(R_{+}\right)}+\|\hat{\phi}\|_{C\left(R_{+}\right)},
$$

we arrive at the following result.

Lemma 2.2. Under the hypothesis of Theorem 1.1 any solution $v$ of problem (1.1), (1.2) satisfies the inequality

$$
\|v\|_{C\left(R_{+}\right)} \leq\|\phi\|_{C(-\eta, 0)}\left[\left(1-\psi_{0} \chi_{0}\left\|V_{M}\right\|_{C\left(R_{+}\right)}\right)^{-1}\left(1+\psi_{0}\right)\left\|V_{M}\right\|_{C\left(R_{+}\right)} \chi_{0}+1\right] .
$$

For an $\epsilon>0$ put

$$
M_{\epsilon}(t):=\sum_{k=0}^{m} B_{k}(t) e^{\epsilon h_{k}(t)}+\int_{0}^{1} B(t, \tau) e^{\epsilon h_{0}(\tau)} d \tau+\epsilon I
$$

and

$$
\left(V_{M, \epsilon} f\right)(t)=\int_{0}^{t} U_{\epsilon}(t, s) f(s) d s \quad\left(f \in C\left(R_{+}\right)\right),
$$

where $U_{\epsilon}(t, s)$ is the evolution operator of the differential equation $z^{\prime}(t)=M_{\epsilon}(t) z(t)$.

Lemma 2.3. If $V_{M}$ is bounded in $C\left(R_{+}\right)$, then for all sufficiently small $\epsilon>0 V_{M, \epsilon}$ is also bounded in $C\left(R_{+}\right)$and $\left\|V_{M, \epsilon}\right\|_{C\left(R_{+}\right)} \rightarrow\|V\|_{C\left(R_{+}\right)}$as $\epsilon \downarrow 0$.

Proof. Define the operators

$$
(R z)(t)=z^{\prime}(t)-M(t) z(t),\left(R_{\epsilon} z\right)(t)=z^{\prime}(t)-M_{\epsilon}(t) z(t)
$$

with the joint domain $D_{0}=\left\{z \in C\left(R_{+}\right): z^{\prime} \in C\left(R_{+}\right), z(0)=0\right\}$. By the Variation of Constants formula we have $R^{-1}=V_{M}$ and $R_{\epsilon}^{-1}=V_{M, \epsilon}$. Clearly, $\left\|M_{\epsilon}(.)-M(.)\right\|_{C\left(R_{+}\right)} \rightarrow 0(\epsilon \rightarrow 0)$. Hence, $\left\|R_{\epsilon}-R\right\|_{C\left(R_{+}\right)}=\left\|M_{\epsilon}(.)-M(.)\right\|_{C\left(R_{+}\right)} \rightarrow$ 0. Obviously

$$
R_{\epsilon}=R+M_{\epsilon}(.)-M(.)=R\left(I+R^{-1}\left(M_{\epsilon}(.)-M(.)\right)\right) .
$$

$R$ is boundedly invertible, since $R^{-1}=V_{M}$ is bounded. So if $\left\|R^{-1}\left(M_{\epsilon}(.)-M(.)\right)\right\|_{C\left(R_{+}\right)}<1$, then $R_{\epsilon}$ is boundedly invertible. Since $R_{\epsilon}^{-1}=V_{M, \epsilon}$, this proves the lemma.

Proof of Theorem 1.1: Substituting

$$
v(t)=y_{\epsilon}(t) e^{-\epsilon t}(\epsilon>0)
$$

into (1.1), we obtain the equation

$$
y_{\epsilon}^{\prime}(t)=\left(E_{\epsilon} y_{\epsilon}\right)(t)
$$

where

$$
\begin{gathered}
\left(E_{\epsilon} u\right)(t)=\sum_{k=1}^{m} B_{k}(t) e^{\epsilon h_{k}(t)} u\left(t-h_{k}(t)\right)+\int_{0}^{1} B(t, \tau) e^{\epsilon h_{0}(t)} u\left(t-h_{0}(\tau)\right) d \tau+\epsilon I \\
\left(u \in C\left(R_{\eta}\right) ; t \geq 0\right) .
\end{gathered}
$$

We have

$$
\sup _{t \geq 0}\left(\sum_{k=1}^{m} e^{\epsilon h_{k}(t)}\left\|B_{k}(t)\right\|+\int_{0}^{1} e^{\epsilon h_{0}(\tau)}\|B(t, \tau)\| d \tau\right)+\epsilon I \rightarrow \chi_{0}
$$


as $\epsilon \rightarrow 0$. So according to Lemma 2.3 conditions (1.3) and (1.5) hold for all sufficiently small $\epsilon$ with $V_{M, \epsilon}$ instead of $V_{M}$ and $E_{\epsilon}$ instead of $E$. Besides, due to Lemmas 2.2 solutions of (2.11) are bounded. Now (2.10) yields the exponential stability. As claimed.

\section{Equations with dissipative operators}

We will say that $A \in \mathcal{B}(X)$ is dissipative if $\|I+A \delta\|<1$ for all sufficiently small $\delta>0$. In this section we consider (1.1), assuming that $M(t)$ is dissipative for sufficiently large $t$. To this end introduce the operator products

$$
\begin{gathered}
\prod_{1 \leq k \leq m}^{\leftarrow}\left(I+M\left(t_{k}^{(m)}\right) \delta_{k}\right):= \\
\left(I+M\left(t_{m}^{(m)}\right) \delta_{m}\right)\left(I+M\left(t_{m-1}^{(m)}\right) \delta_{m-1}\right) \cdots\left(I+M\left(t_{1}^{(m)}\right) \delta_{1}\right)
\end{gathered}
$$

where

$$
s=t_{1}^{(m)}<t_{2}^{(m)}<\ldots<t_{m}^{(m)}=t \text { and } \delta_{k}=t_{k}^{(m)}-t_{k-1}^{(m)}(k=1, \ldots, m) .
$$

I.e., the arrow over the symbol of the product means that the indexes of the co-factors increase from right to left. The strong limit of these products as $\max _{k} \delta_{k} \rightarrow 0$ (if it exists) is called the left multiplicative integral and is denoted by $\int_{[s, t]}^{\leftarrow}\left(I+M\left(s_{1}\right) d s_{1}\right)$. As it is well-known,

$$
U(t, s)=\int_{[s, t]}^{\overleftarrow{ }}\left(I+M\left(s_{1}\right) d s_{1}\right)
$$

This equality is proved in [9, Chapter 1].

Lemma 3.1. Let there be a real Riemann-integrable function function $v(t)$, such that

$$
\|I+M(t) \delta\| \leq 1+v(t) \delta+o(\delta)(t \geq 0)
$$

for all sufficiently small $\delta>0$. Then

$$
\|U(t, s)\| \leq \exp \left[\int_{s}^{t} v\left(s_{1}\right) d s_{1}\right] \quad(t \geq s \geq 0) .
$$

Proof. We have

$$
\left\|\prod_{1 \leq k \leq m}^{\leftarrow} e^{\left.M\left(t_{k}^{(m)}\right)\right) \delta_{k}}\right\| \leq \prod_{k=1}^{m} e^{v\left(t_{k}^{(m)}\right) \delta_{k}}=\exp \left[\sum_{k=1}^{m} v\left(t_{k}^{(m)}\right) \delta_{k}\right]
$$

The passage to the limit as $m \rightarrow \infty$ and representation (3.1) give the required estimate.

Assume that

$$
\theta_{M}:=\sup _{t \geq 0} \int_{0}^{t} \exp \left[\int_{s}^{t} v\left(s_{1}\right) d s_{1}\right] d s<\infty .
$$

Then due to Lemma $3.1\left\|V_{M}\right\|_{C\left(R_{+}\right)}=\left\|V_{M}\right\|_{C\left(R_{+}, x\right)} \leq \theta_{M}$, provided (3.2) holds.

Now Theorem 1.1 yields.

Corollary 3.2. Let the conditions (1.3), (3.2), (3.3) and $\psi_{0} \chi_{0} \theta_{M}<1$ hold. Then (1.1) is exponentially stable. 
Let $X=\mathcal{H}$ be a Hilbert space and $\Lambda\left(M_{R}(t)\right)=\sup \sigma\left(M_{R}(t)\right)$, where $M_{R}(t)=\frac{1}{2}\left(M(t)+M^{*}(t)\right)$ and the asterisk means the adjointness. Since

$$
\begin{gathered}
\|(I+M(t) \delta) h\|^{2}=\|(I+M(t) \delta) h\|^{2}=\left\|\left(I+2 M_{R}(t) \delta+M^{\star}(t) M(t) \delta^{2}\right) h\right\| \\
\leq 1+2 \Lambda\left(M_{R}(t)\right) \delta+o(\delta) \quad(h \in \mathcal{H},\|h\|=1),
\end{gathered}
$$

we can take $v(t)=\Lambda\left(M_{R}(t)\right)$. Hence we arrive at the Wintner inequality

$$
\|U(t, s)\| \leq \exp \left[\int_{s}^{t} \Lambda\left(M_{R}\left(s_{1}\right)\right) d s_{1}\right] \quad(t \geq s \geq 0),
$$

cf [6, Theorem III.4.7]. So in the case $\mathcal{X}=\mathcal{H}$ Corollary 3.2 can be applied with

$$
\theta_{M}=\sup _{t \geq 0} \int_{0}^{t} \exp \left[\int_{s}^{t} \Lambda\left(M_{R}\left(s_{1}\right)\right) d s_{1}\right] d s
$$

provided $\theta_{M}<\infty$.

\section{Equations in a Banach space with the generalized Lipschitz property}

In this section we consider equation (1.1), assuming that $M(t)$ satisfies the generalized Lipschitz condition

$$
\|M(t)-M(\tau)\| \leq a(|t-\tau|) \quad(t, \tau \geq 0),
$$

where $a(t)$ is a non-negative piece-wise continuous function defined on $[0, \infty)$ and $a(0)=0$. A particular case of (4.1) is the traditional Lipschitz condition

$$
\|M(t)-M(\tau)\| \leq q_{0}|t-\tau|\left(q_{0}=\text { const }>0 ; t, \tau \geq 0\right) .
$$

In addition to (4.1) suppose that there is a positive integrable on $[0, \infty)$ function $p(t)$ independent of $s$, such that

$$
\|\exp [M(s) t]\| \leq p(t) \quad(t, s \geq 0) \text { and } J_{0}:=\int_{0}^{\infty} p(t) d t<\infty .
$$

Lemma 4.1. Let the conditions (4.1), (4.2) and

$$
\zeta_{0}:=\int_{0}^{\infty} a(s) p(s) d s<1
$$

hold. Then a solution $u(t)$ to the non-homogeneous problem

$$
\frac{d u(t)}{d t}=M(t) u(t)+f(t), u(0)=0 \quad\left(f \in C\left(R_{+}, X\right)\right)
$$

satisfies the inequality

$$
\|u\|_{C\left(R_{+}, x\right)} \leq \frac{J_{0}\|f\|_{C\left(R_{+}, x\right)}}{1-\zeta_{0}}
$$

Proof. Rewrite (4.4) as

$$
\frac{d u(t)}{d t}=M(\tau) u(t)+[M(t)-M(\tau)] u(t)+f(t)
$$


with an arbitrary fixed $\tau \geq 0$. So problem (4.4) is equivalent to the equation

$$
u(t)=\int_{0}^{t} \exp [M(\tau)(t-s)]([M(s)-M(\tau)] u(s)+f(s)) d s .
$$

Hence,

$$
\|u(t)\| \leq \int_{0}^{t}\|\exp [M(\tau)(t-s)]\|(\|M(s)-M(\tau)\|\|u(s)\|+\|f(s)\|) d s .
$$

According to (4.1) and (4.2),

$$
\|u(t)\| \leq \int_{0}^{t} p(t-s)(a(|s-\tau|)\|u(s)\|+\|f(s)\|) d s .
$$

Taking $\tau=t$, we obtain

$$
\|u(t)\| \leq \int_{0}^{t} p(t-s)(a(t-s)\|u(s)\|+\|f(s)\|) d s .
$$

Hence for any positive finite $T$, with $\sup _{t \leq T}\|u(t)\|=|u|_{T}$ we have

$$
|u|_{T} \leq|u|_{T} \int_{0}^{T} p\left(t_{1}\right) a\left(t_{1}\right) d t_{1}+\int_{0}^{T} p\left(t_{1}\right) d t_{1}\|f\|_{C\left(R_{+}\right)}
$$

Consequently, $|u|_{T} \leq|u|_{T} \zeta_{0}+J_{0}\|f\|_{C\left(R_{+}\right)}$. According to (4.3) we get

$$
|u|_{T} \leq\left(1-\zeta_{0}\right)^{-1} J_{0}\|f\|_{C\left(R_{+}\right)} .
$$

Extending this result to all $T \geq 0$, we prove the lemma.

From this lemma it follows.

Corollary 4.2. Let conditions (4.1) and (4.2) hold. Then

$$
\left\|V_{M}\right\|_{C\left(R_{+}\right)} \leq \frac{J_{0}}{1-\zeta_{0}} .
$$

Due to this corollary condition (1.5) under the hypothesis of the previous lemma is provided by the inequality $\chi_{0} \psi_{0} \frac{J_{0}}{\left(1-\zeta_{0}\right)}<1$. Or

$$
\chi_{0} \psi_{0} J_{0}+\zeta_{0}<1
$$

Now Theorem 1.1 yields.

Corollary 4.3. Let the conditions (1.3), (4.1), (4.2) and (4.5) hold. Then equation (1.1) is exponentially stable.

\section{Equations with differentiable coefficients in a Hilbert space}

\subsection{Statement of the result}

In this section $X=\mathcal{H}$ is a separable Hilbert space with a scalar product (., .). By $\mathcal{B}(\mathcal{H})$ the set of all linear bounded operators acting in $\mathcal{H}$ is denoted. 
Consider equation (1.1) in $\mathcal{H}$, assuming that operator $M(t)$ defined in Section 1 is uniformly bounded on $R_{+}$, has a measurable strong derivative uniformly bounded on $R_{+}$and

$$
\alpha_{M}:=\sup _{t \geq 0} \alpha(M(t))=\sup _{t \geq 0} \sup \operatorname{Re} \sigma(M(t))<0 .
$$

Introduce quantity

$$
q(t):=2 \int_{0}^{\infty}\left\|e^{M(t) s}\right\|^{2} d s .
$$

By the classical Dunford-Cauchy formula for operator functions (see for instance [6, Ch. 1]),

$$
e^{M(t) t}=\frac{1}{2 \pi i} \int_{C} e^{t z}(I z-M(t))^{-1} d z,
$$

where $C$ is a Jordan contour surrounding $\sigma(M(t))$. Hence, for each $t \geq 0$ we have

$$
\left\|e^{M(t) s}\right\| \leq \mathrm{const} e^{s\left(\alpha_{M}+\epsilon\right)}(\epsilon>0, s \geq 0)
$$

and therefore $q(t)<\infty$. It is assumed that

$$
\sup _{t \geq 0} q^{2}(t)\left\|M^{\prime}(t)\right\|<2
$$

and

$$
\gamma_{M}:=\sup _{t \geq 0} \sqrt{\left|\lambda_{\inf }\left(M_{R}(t)\right)\right|} \int_{0}^{t} \sqrt{q(s)} \exp \left[-\int_{s}^{t} \frac{\left(1-\frac{1}{2} q^{2}\left(t_{1}\right) \| M^{\prime}\left(t_{1}\right)||\right)}{q\left(t_{1}\right)} d t_{1}\right] d s<\infty,
$$

where $\lambda_{\text {inf }}\left(M_{R}(t)\right):=\inf \sigma\left(M_{R}(t)\right)$. Recall that $M_{R}(t)=\frac{1}{2}\left(M(t)+M^{\star}(t)\right)$.

Theorem 5.1. Let the conditions (1.3), (5.1), (5.2) and

$$
\chi_{0} \gamma_{M} \psi_{0}<1
$$

hold. Then (1.1) is exponentially stable.

The proof of this theorem is presented in the next subsection.

\subsection{Proof of Theorem 5.1}

An operator $A_{0} \in \mathcal{B}(\mathcal{H})$ is said to be stable if $\alpha\left(A_{0}\right)=$ sup $\operatorname{Re} \sigma\left(A_{0}\right)<0$. As is well known the Lyapunov equation

$$
A_{0}^{\star} Y+Y A_{0}=-2 I
$$

with a stable operator $A_{0}$ has a solution $Y$ which is represented as

$$
Y=2 \int_{0}^{\infty} e^{A_{0}^{\star} s} e^{A_{0} s} d s
$$

cf. [6, Section I.4.4]. Put $y(t)=e^{A_{0} t} z(z \in \mathcal{H})$. Then $y^{\prime}(t)=A_{0} y(t)$, and

$$
\frac{d(y(t), y(t))}{d t}=\left(\left(A_{0}+A_{0}^{\star}\right) y(t), y(t)\right) \text {. }
$$

With $\operatorname{Re} A_{0}=\left(A_{0}+A_{0}^{\star}\right) / 2$ and $\lambda_{\text {inf }}\left(\operatorname{Re} A_{0}\right)=\inf \sigma\left(\operatorname{Re} A_{0}\right)$, we conclude that

$$
\frac{d(y(t), y(t))}{d t} \geq 2 \lambda_{\text {inf }}\left(\operatorname{Re} A_{0}\right)(y(t), y(t)) \text { and therefore }\left\|e^{A_{0} t} z\right\| \geq e^{t \lambda_{\text {inf }}\left(\operatorname{Re} A_{0}\right)}\|z\|(t \geq 0)
$$


Since $A_{0}$ is stable, $\left\|e^{A_{0} t} z\right\| \rightarrow 0$ and therefore $e^{t \lambda_{\text {inf }}\left(\operatorname{Re} A_{0}\right)} \rightarrow 0(t \rightarrow \infty)$. Hence it follows that $\lambda_{\text {inf }}\left(\operatorname{Re} A_{0}\right)<0$. We thus get

$$
(Y z, z)=2 \int_{0}^{\infty}\left(e^{A_{0}^{*} s} e^{A_{0} s} z, z\right) d s \geq 2 \int_{0}^{\infty} e^{2 \lambda_{\text {inf }}\left(\operatorname{Re} A_{0}\right) s} d s\|z\|^{2}=\frac{\|z\|^{2}}{\left|\lambda_{\inf }\left(\operatorname{Re} A_{0}\right)\right|}(z \in \mathcal{H}) .
$$

Furthermore, according to (5.4) the operator

$$
Q(t):=2 \int_{0}^{\infty} e^{M^{*}(t) s} e^{M(t) s} d s
$$

is a unique solution of the equation

$$
M^{\star}(t) Q(t)+Q(t) M(t)=-2 I \quad(t \geq 0) .
$$

Clearly, $\|Q(t)\| \leq q(t)$. Due to (5.5)

$$
(Q(t) z, z) \geq \frac{\|z\|^{2}}{\left|\lambda_{\text {inf }}\left(M_{R}(t)\right)\right|}(z \in \mathcal{H}, t \geq 0) .
$$

Lemma 5.2. Let condition (1.3) hold and $M(t)$ be strongly differentiable. Then $Q(t)$ is strongly differentiable and $\left\|Q^{\prime}(t)\right\| \leq q^{2}(t)\left\|M^{\prime}(t)\right\|$.

For the proof see Lemma 2.1 from [13].

Lemma 5.3. Let $M(t)$ be strongly differentiable. Then

$$
\frac{d}{d t}(Q(t) u(t), u(t)) \leq\left(-2+\left\|Q^{\prime}(t)\right\|\right)(u(t), u(t))
$$

for any solution to (1.4).

Proof. Multiplying equation (1.4) by $Q(t)$ and doing the scalar product, we can write

$$
\left(Q(t) u^{\prime}(t), u(t)\right)=(Q(t) M(t) u(t), u(t)) .
$$

Since

$$
\frac{d}{d t}(Q(t) u(t), u(t))=\left(Q(t) u^{\prime}(t), u(t)\right)+\left(u(t), Q(t) u^{\prime}(t)\right)+\left(Q^{\prime}(t) u(t), u(t)\right),
$$

in view of (5.6) we get

$$
\begin{gathered}
\frac{d}{d t}(Q(t) u(t), u(t))=(Q(t) M(t) u(t), u(t))+(u(t), Q(t) M(t) u(t))+\left(Q^{\prime}(t) u(t), u(t)\right) \\
=\left(\left(Q(t) M(t)+M^{\star}(t) Q(t)\right) u(t), u(t)\right)+\left(Q^{\prime}(t) u(t), u(t)\right) \\
=-2(u(t), u(t))+\left(Q^{\prime}(t) u(t), u(t)\right) .
\end{gathered}
$$

Hence, we get the required result.

Let

$$
\sup _{t \geq 0}\left\|Q^{\prime}(t)\right\|<2
$$

Then according to the previous lemma

$$
\frac{d}{d t}(Q(t) u(t), u(t))=\left(\left(-2 I+Q^{\prime}(t)\right) u(t), u(t)\right) \leq-\frac{\left(2-\left\|Q^{\prime}(t)\right\|\right)}{\|Q(t)\|}(Q(t) u(t), u(t)) .
$$

Solving this inequality and taking into account that $\|Q(t)\| \leq q(t)$, we obtain

$$
(Q(t) u(t), u(t)) \leq(Q(s) u(s), u(s)) \exp \left[-\int_{s}^{t} \frac{\left(2-\left\|Q^{\prime}\left(t_{1}\right)\right\|\right)}{q\left(t_{1}\right)} d t_{1}\right]
$$


Now (5.7) implies

$$
\|u(t)\|^{2} \leq\left|\lambda_{\text {inf }}\left(M_{R}(t)\right)\right| q(s)\|u(s)\|^{2} \exp \left[-\int_{s}^{t} \frac{\left(2-\left\|Q^{\prime}\left(t_{1}\right)\right\|\right)}{q\left(t_{1}\right)} d t_{1}\right] .
$$

Making use of Lemma 5.2, we arrive at

Lemma 5.4. Let conditions (5.1) and (5.2) hold. Then the evolution operator of (1.4) satisfies the inequality

$$
\|U(t, s)\|^{2} \leq\left|\lambda_{\mathrm{inf}}\left(M_{R}(t)\right)\right| q(s) \exp \left[-\int_{s}^{t} \frac{\left(2-q^{2}\left(t_{1}\right)\left\|M^{\prime}\left(t_{1}\right)\right\|\right)}{q\left(t_{1}\right)} d t_{1}\right](t \geq s \geq 0) .
$$

Proof of Theorem 5.1: Obviously,

$$
\|V\|_{C\left(R_{+}\right)} \leq \sup _{t} \int_{0}^{t}\|U(t, s)\| d s .
$$

Hence, due to Lemma $5.4\|V\|_{C\left(R_{+}\right)} \leq \gamma_{M}$. Now Theorem 1.1 yields the required result.

\section{Integro-differential equations with delays}

Our main object in this section is the equation

$$
\frac{\partial u(t, x)}{\partial t}=c(t, x) u\left(t-h_{1}(t), x\right)+\int_{0}^{x} k\left(t, x, x_{1}\right) u\left(t-h_{2}(t), x_{1}\right) d x_{1} \quad(t>0 ; 0 \leq x \leq 1),
$$

where $c(.,):.[0, \infty) \times[0,1] \rightarrow \mathbb{R}$ is continuous in $t$ and piece-wise continuous in $x ; k(., .,):.[0, \infty) \times[0,1]^{2} \rightarrow$ $\mathbb{R}$ has the property: the integral $\int_{0}^{1}\left|k\left(t, x, x_{1}\right)\right| d x_{1}$ is continuous in $t \geq 0$.

We consider equation $(6.1)$ in the space $C(0,1)$ of scalar continuous functions defined on $[0,1]$ with the sup-norm. Equation (6.1) has the form (1.1) with $m=2 ; h_{1}(t), h_{2}(t)$ are the same as above; $B(t, \tau)=0$,

$$
\left(B_{1}(t) z\right)(x)=c(t, x) z(x),\left(B_{2}(t) z\right)(x)=\int_{0}^{1} k\left(t, x, x_{1}\right) z\left(x_{1}\right) d x_{1} \quad(z \in C(0,1)),
$$

and $M(t)=B_{1}(t)+B_{2}(t)$. Condition (1.3) takes the form

$$
\chi_{1}=\sup _{t \geq 0}\left(\sup _{x}|c(t, x)|+\left\|B_{2}(t)\right\|\right)<\infty .
$$

So in the considered case $\chi_{0}=\chi_{1}$ and $\psi_{0}=\psi_{1}$, where

$$
\psi_{1}=\sup _{t \geq 0}\left(h_{1}(t) \sup _{x}|c(t, x)|+h_{2}(t)\left\|B_{2}(t)\right\|\right) .
$$

Condition (3.2) takes the form

$$
\|I+M(t) \delta\|=\max _{x}\left|z(x)+c(t, x) z(x) \delta+\left(B_{2}(t) z\right)(x) \delta\right| \leq 1+v(t) \delta+o(\delta)(\|z\|=1) .
$$

For example, if

$$
c(t, x)+\left\|B_{2}(t)\right\| \leq-v_{1}(t) \quad(0 \leq x \leq 1, t \geq 0),
$$

where $v_{1}(t)$ is a positive continuous function, then (6.3) holds with $v(t)=-v_{1}(t)$. If

$$
\Lambda_{1}:=\sup _{t \geq 0} v(t)<0
$$


then according to (3.3)

$$
\theta_{M}=\sup _{t \geq 0} \int_{0}^{t} \exp \left[\int_{s}^{t} v\left(s_{1}\right) d s_{1}\right] d s \leq \sup _{t \geq 0} \int_{0}^{t} \exp \left[(t-s) \Lambda_{1} \mid\right] d s=\frac{1}{\left|\Lambda_{1}\right|} .
$$

Now Corollary 3.2 implies.

Corollary 6.1. Let the conditions (6.2)-(6.4) and $\psi_{1} \chi_{1}<\left|\Lambda_{1}\right|$ hold. Then (6.1) is exponentially stable.

About other approaches to integro-differential equations see $[1-4,17,19]$.

Conflict of interest: The author states that there is no conflict of interest.

Data Availability Statement: Data sharing is not applicable to this article as no datasets were generated or analysed during the current study.

\section{References}

[1] J. Appel, A. Kalitvin and P. Zabreiko, Partial integral operators and integrodifferential equations, Marcel Dekker, New York, 2000.

[2] H. Jr. Cassago and C. Corduneanu, The ultimate behavior for certain nonlinear integro-differential equations. J. Integral Equations 9 (1985), no. 2, 113-124.

[3] Chuhu Jin and Jiaowan Luo, Stability of an integro-differential equation, Computers and Mathematics with Applications, 57 (2009) 1080-1088.

[4] C. Corduneanu, Periodic solutions of certain integro-differential systems. Libertas Math. 7 (1987), 149-154.

[5] C. Corduneanu, Yizeng Li and M. Mahdavi, Functional differential equations. Advances and applications. Pure and Applied Mathematics (Hoboken). John Wiley \& Sons, Inc., Hoboken, NJ, 2016.

[6] Yu L. Daleckii, and M.G. Krein, Stability of solutions of differential equations in Banach space, Amer. Math. Soc., Providence, R. I. 1974.

[7] R. Datta, B. Bhattacharya and A. Chakrabarti, On improved delay-range-dependent stability condition for linear systems with time-varying delay via Wirtinger inequality. Int. J. Dyn. Control 6 (2018), no. 4, 1745-1754.

[8] R. Dey, G. Martinez and C. Juan, Improved delay-range-dependent stability analysis for uncertain retarded systems based on affine Wirtinger-inequality. Internat. J. Robust Nonlinear Control 27 (2017), no. 16, 3028-3042.

[9] J.D. Dollard and Ch. N. Friedman, Product integration with applications to differential equations. Encyclopedia of Mathematics and its applications; v.10., London, Addison-Wesley Publ. Company, 1979.

[10] E. Fridman, and Orlov, Exponential stability of linear distributed parameter systems with time-varying delays. Automatica, 45, (2009) 194-201.

[11] M.I. Gil', Stability of abstract nonlinear nonautonomous differential-delay equations with unbounded history-responsive operators. Journal of Mathematical Analysis and Applications, 308, (2005) 140-158.

[12] M.I. Gil', On stability of linear Barbashin type integro-differential equations, Mathematical Problems in Engineering, 2015, Article ID 962565, (2015), 5 pages.

[13] M.I. Gil', Stability of linear equations with differentiable operators in a Hilbert space, IMA Journal of Mathematical Control and Information, 37 (2020), no. 1, 19-26.

[14] K. Ikeda, T. Azuma, and K. Uchida, Infinite-dimensional LMI approach to analysis and synthesis for linear time-delay systems. Special issue on advances in analysis and control of time-delay systems. Kybernetika (Prague), 37, no 1(2001) 505-520.

[15] Tao Li, Wei Qian Ting, Wang and Shumin Fei, Further results on delay-dependent absolute and robust stability for time-delay Lur'e system. Internat. J. Robust Nonlinear Control 24 (2014), no. 18, 3300-3316.

[16] Y.P. Luo, and F.Q. Deng, LMI-based approach of robust control for uncertain distributed parameter control systems with time-delay. Control Theory and Applications, 23, (2006) 318-324.

[17] J. Vanualailai and S. Nakagiri, Stability of a system of Volterra integro-differential equations, J. Math. Anal. Appl. 281 (2003) 602-619

[18] L. Wang and Y. Wang, LMI-based approach of global exponential robust stability for a class of uncertain distributed parameter control systems with time-varying delays. Journal of Vibration and Control, 15, (2009) 1173-1185.

[19] Van Zhang and B. Zhang, Construction of Liapunov functionals for linear Volterra integrodifferential equations and stability of delay systems, Electron. J. Qual. Theory Differ. Equ. 30 (2000) 1-17. 